\title{
Origin of Stars of Planetary Systems
}

Chairman \& Editor: A. Brahic 


\section{ORIGIN OF STARS AND PLANETARY SYSTEMS}

Joint Discussion with Commissions 7, 15, 16, 20, 22, 28, 33, 35, 37, 45, 48, and 51.

Organizing Committee: A.H. Batten, A. Brahic (Chairman), M. Golay, G.L. Harris, A. Henrard, C.S.L. Keay, A. Maeder, G. Marx, M. Mayor, R.A. Sunyaev, G.A. Tamman, R. West.

A large attendance participated to this meeting. It is a pity a few invited speakers could not come to the meeting for private reasons. Several participants expressed their satisfaction concerning the inter-disciplinary exchanges during this meeting. Specialists of the solar system and astronomers from other areas than members of the planetary community have in fact very few opportunities to meet. Such meetings should be more often organized. The program of the oral presentations of the Joint Discussion Meeting is given below:

- Planetary Atmospheres and Chemical Constraints

- Comets and Solar System Formation

- Planetary Rings and Dynamical Constraints

- From Planetoids to Planets

- Search for Extra Solar Planets from Space

- The B Pictoris Disk

- Circumstellar Disks and Star Formation

- Circumstellar Environnement of Young Stellar Objects

- Magnetic Activity around T Tauri Stars

- Effects of Interactions Involving Protostellar Disks
T. Owen

A.C. Levasseur-Regourd

A. Brahic

P. Barge and R. Pellat

R. Terrile

B. Smith

L. Hartmann and M. Gomez

T. Montmerle

T. Montmerle

R.B. Larson and C.H. Heller

The articles corresponding to the communications of Dr. T. Montmerle are published in "The Physics of Star Formation and Early Stellar Evolution" (ed. C. Lada and N. Kylafis), N.A.T.O. A.S.I., Kluwer, 1991 and in "Nonisotropic and variable outflows from stars" (ed. L. Drissen, C. Leitherer, and A. Notta), A.S.P. Conference Series (in press).

A large part of the results presented by Dr. R.B. Larson and C.H. Heller are printed in Dr. C.H. Heller thesis "Encounters with Protostellar Disks" which was submitted in December 1991 in the Department of Astronomy of Yale University (copies should be available from University Microfilms).

A book entitled "Protostars and Planets III" (E. Levy and J. Lunine eds., University of Arizona Press, Tucson, 1992) will be soon published. It contains many upto-date information. 